\title{
MICROCANTILEVER SENSORS
}

T. Thundat*, P. I. Oden ${ }^{*}$, P. G. Dasktos* $\ddagger$, G. Y. Chen*ł and R. J. Warmack*

*Health Sciences Research Division, Oak Ridge National Laboratory, Oak Ridge, TN 37831-6123

+Oak Ridge Associated Universities, Oak Ridge, TN 37830

$\ddagger$ Department of Physics and Astronomy, University of Tennessee, Knoxville, TN 37996

\begin{abstract}
Novel sensors based on bending and resonance frequency changes of microcantilevers are discussed. The adsorption-induced resonance frequency changes of microcantilevers can be due to a combination of mass loading and change of spring constant resulting from adsorption of chemicals on the surface. Cantilevers also undergo static bending due to adsorption-induced differential surface stress if the adsorption is confined to one surface. Hence cantilever deflection as well as resonance frequency change can be used as the basis for development of novel chemical sensors.
\end{abstract}

\section{INTRODUCTION}

Recently it has become clear that microcantilevers, such as those designed for atomic force microscopy (AFM) [1], can be used for a variety of sensor applications [2-5]. For example, microcantilevers that are metal coated on one side are very sensitive to temperature, and undergo static bending as a result of slight variation in temperature due to the bimetallic effect. Recently we have reported that bending of aluminum coated microcantilevers can be influenced by changes in relative humidity and that the sensitivity of this effect can be increased by coating the cantilevers with hygroscopic materials [4]. More importantly, the concept can be extended to detection of numerous other chemical vapors.

Changes in cantilever resonance frequency provide a direct measure of the mass of adsorbed vapors if the spring constant remains fixed. However, in many cases the spring constant changes as a result of vapor adsorption, significantly complicating interpretation of experimental results. In this paper we show that simultaneous measurement of bending and resonance frequency change can be used to decouple the effects of mass change and spring constant variation in the interpretation of resonance frequency changes.

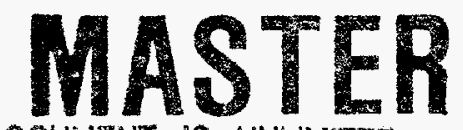

DISTREUTON OF THS DOCUENT IS UWMITED DLC
"The submitted manuscript has been authored by a contractor of the U.S. Government under contract no DE-AC05-960R22464. Accordingly, the U.S. Government retains a non exclusive, royalty-free license to publish or reproduce the published form of this contribution or allow other to do so, for U.S. Government Purposes." 


\section{EXPERIMENTAL}

Commercially available, "V"-shaped silicon cantilevers $(200-\mu \mathrm{m}$ long, $0.06-\mathrm{N} / \mathrm{m}$ or 0.09 $\mathrm{N} / \mathrm{m}$ spring constant Ultralevers, Park Scientific Instruments, Inc.) were used in this study. One set of cantilevers was coated on one side with gelatin for relative humidity investigations. The thickness of the coating was estimated by determining the change in resonance frequency for the coated cantilever. In addition, a second set of cantilevers was coated on a single side with an evaporatively deposited gold film to allow evaluation of the effects of mercury adsorption.

The deflection and resonance frequency of coated cantilevers were measured using the position sensitive detector of a Multi-Mode Nanoscope III. Tip deflection was measured by monitoring the normalized error voltage $\left(\mathbf{V}_{\text {error }}\right)$ between the top and bottom segments of the Nanoscope position sensitive detector. Adsorption of water vapor on the gelatin coated cantilevers was controlled by placing the AFM head in a chamber purged with humidified nitrogen gas; the atmosphere in the chamber was monitored using a hygrometer. Adsorption of mercury vapor on gold-coated cantilevers was achieved by placing a Knudsen cell containing mercury in the chamber housing the AFM head [5].

\section{RESULTS AND DISCUSSION}

The resonance frequency, $v$, of an oscillating cantilever can be expressed as

$$
v=\frac{1}{2 \pi} \sqrt{\frac{K}{m^{*}}}
$$

where $K$ is the spring constant and $m^{*}$ is the effective mass of the cantilever. Note that $m^{*}=$ $\boldsymbol{n} \boldsymbol{m}_{b}$, where $\boldsymbol{m}_{b}$ is the mass of the cantilever beam and the value of $\boldsymbol{n}$ is either 0.14 for a 0.06 $\mathrm{N} / \mathrm{m}$ or 0.18 for a $0.03 \mathrm{~N} / \mathrm{m}$ " $\mathrm{V}$ " shaped silicon nitride cantilever or 0.24 for a rectangular cantilever. When adsorbates are deposited uniformly on the cantilever surface, the resultant mass change, $\delta m$, can be calculated fromvariation in resonance frequency. This interpretation of frequency shift assumes that changes in spring constant, $\delta K$, are negligible. However, from Eq. (1) it is clear that changes in resonance frequency can result from both mass change or variation in $K$. 
Fig. 1a shows the variation in resonance frequency of a gelatin coated cantilever due to exposure to water vapor, while Fig. $1 \mathrm{~b}$ shows the deflection as a function of relative humidity during the same exposure. The increase in resonance frequency as humidity is increased is counter to that anticipated due to mass loading, suggesting that a change in spring constant is also occurring. The nearly linear response in both figures indicates that the magnitude of $\delta m$ and $\delta K$ are small relative to the initial values.

From this and other similar experiments it is clear that when molecules adsorb on a cantilever surface, the resonance frequency of the cantilever changes due to mass loading. In addition to resonance frequency change, deflection (bending) may change due to adsorption induced differential surface stress, $\delta$ s. Taking into account the boundary conditions of a cantilever, the displacement of the cantilever, $z$ can be written as

$$
z=\left[\frac{3(1-v) L^{2}}{t^{2} E}\right] \delta s
$$

where $L$ is the length of the cantilever, and $v$ is Poisson's ratio.

Surface stress can also effect the spring constant of the cantilever. To account for this, Eq. (1) can be modified as

$$
v_{2}=\frac{1}{2 \pi} \sqrt{\frac{K+8 K}{m^{*}+n \delta m}}
$$

where the initial resonance frequency $v_{1}$ changes to $v_{2}$ due to adsorption. In this equation $K$ changes to $K+\delta K$ as a result of adsorption induced surface stress while $m^{*}$ changes to $m^{*}+n \delta m$ due to mass loading. $\delta K$ is proportional to $\delta s[6]$.

By designing cantilevers with localized adsorption areas at the terminal end of the cantilever (end loading), the contribution from differential surface stress can be minimized and changes in resonance frequency can be entirely attributed to mass loading. Figure 4 shows the resonance frequency response upon exposure to mercury vapor for a cantilever that was coated with gold over the terminal $43 \mu \mathrm{m}$ from the apex [5]. In contrast to cantilevers coated with gold along their entire length, these cantilevers show negligible bending due to mercury adsorption. It is clear that the frequency response (with a slope opposite to that of fully coated cantilevers) 
in this case is entirely due to mass loading. An adsorption rate of $32.8 \times 10^{-6} \mathrm{~kg} \mathrm{~m}^{-2} \mathrm{~min}^{-1}$ was calculated from the data shown in Fig. 4. The sensitivity of frequency response in Fig. 4 is calculated to be $0.8 \mathrm{pg} / \mathrm{Hz}$.

For a cantilever coated with gold along its entire length $\left(m_{b}=34.6 \mathrm{ng}\right.$, ie, Figs. 2 and 3$)$, the equivalent adsorption rate for mercury is $553 \mathrm{pg} / \mathrm{min}$. As noted earlier, the initial deviation of frequency response from the calculated curve in Fig. 2 is probably due to the time required for the mercury vapor to reach equilibrium in the chamber. A calculation carried out using the data in Fig. 2 shows that $K$ increases at the rate of about $0.001 \mathrm{~N} \mathrm{~m}^{-1} \mathrm{~min}^{-1}$. The frequency responses observed in Figs. 2 and 3 appear to be influenced by both variations in spring constant and mass loading. The resonance frequency response can be explained by competing effects of spring constant variation and mass loading, where $\delta v \propto\left(\frac{\delta R}{K}-\frac{\delta m}{m}\right)$. If the first term dominates the expression, the resonance frequency shifts positively.

Based on the slope of the curve in Fig. 3, it is possible to estimate the sensitivity of the method for mercury detection as $0.6 \mathrm{pg} / \mathrm{mV}$. However, the noise for an equilibrated cantilever is approximately $3 \mathrm{mV}$ at room temperature. Thus, the minimum detectable amount of mercury for the current scheme based on cantilever bending is of the order of several picograms. From Fig. 2, the sensitivity of frequency response can be calculated as $11 \mathrm{pg} / \mathrm{Hz}$ (linear region). The sensitivity of relative humidity (R.H.) detection can be calculated as $0.14 \mathrm{RH} / \mathrm{Hz}$ (Fig. 1a, frequency response) and $0.002 \mathrm{RH} / \mathrm{mV}$ (Fig.1b, cantilever deflection).

\section{CONCLUSIONS}

In summary, the adsorption of molecules on cantilever surfaces can produce bending as well as resonance frequency shifts. By simultaneously measuring bending and resonance frequency shifts it is possible to decouple the influence of mass loading and spring constant variations. The sensitivity of the technique using current equipment is in the picogram range, and it can be used to detect chemisorbed or physisorbed adsorbates.

\section{ACKNOWLEDGEMENTS}

We would like to thank Drs. J. C. Ashley and D. P. Allison for useful discussions. This research was sponsored by the U.S. Department of Energy Office of Health and Environmental 
Research under contract number DE-AC05-96OR22464 with Lockheed Martin Energy Research

Corp. The authors also wish to gratefully acknowledge the support of the ORNL Director's Seed Money Fund.

\section{REFERENCES}

1 S. Akamine, R.C. Barrett, and C.F. Quate, Appl. Phys. Lett. 57, 316 (1990).

2 J.K. Gimzewski, Ch. Gerber, E. Meyer, and R.R. Schlittler, Chem. Phys. Lett., 217, 589 (1994).

3 T. Thundat, R.J. Warmack. G.Y. Chen, and D.P. Allison, Appi. Phys. Lett. 64, 2894 (1994).

4 T. Thundat, G.Y. Chen, E.A. Wachter, D.P. Allison, and R.J. Warmack, Anal. Chem., 67 519 (1995).

5 T.Thundat, E.A. Wachter, S.L. Sharp, and R.J. Warmack, Appl. Phys. Lett., 661695 (1995).

6 G.Y. Chen, T. Thundat, E.A. Wachter, and R.J. Warmack, J. Appl. Phys., 773618 (1995).

\section{FIGURE CAPTIONS}

Fig. 1 (a) Resonance frequency response for a gelatin coated silicon cantilever as a function of relative humidity. (b) Bending (error voltage) under same conditions. The nominal $K$ value of the cantilever was $0.06 \mathrm{~N} / \mathrm{m}$ before gelatin modification. The gelatin film thickness for the cantilever was calculated to be about $23 \mathrm{~nm}$.

Fig. 2 Typical plot of error voltage as a function of mercury vapor exposure time for a gold coated silicon nitride cantilever. Uniform $40 \mathrm{~nm}$ thick layer of gold deposited on one side along entire length of cantilever.

Fig. 3 Resonance frequency response as a function of mercury exposure recorded simultaneously with error voltage shown in Fig. 2. The theoretical curve (solid line) was calculated using Eq. (6). The mass of the cantilever, $m_{b}$, is $34.6 \mathrm{ng}$. Mercury vapor adsorption rate and the rate at which $K$ varies are calculated to be $553 \mathrm{pg} / \mathrm{min}$ and $0.001 \mathrm{~N} / \mathrm{m} / \mathrm{min}$ respectively.

Fig. 4 Resonance frequency response of a cantilever with gold coating only at the final $43 \mu \mathrm{m}$ length near the apex. The nominal initial $K$ value of the cantilever was $0.06 \mathrm{~N} / \mathrm{m}$. 


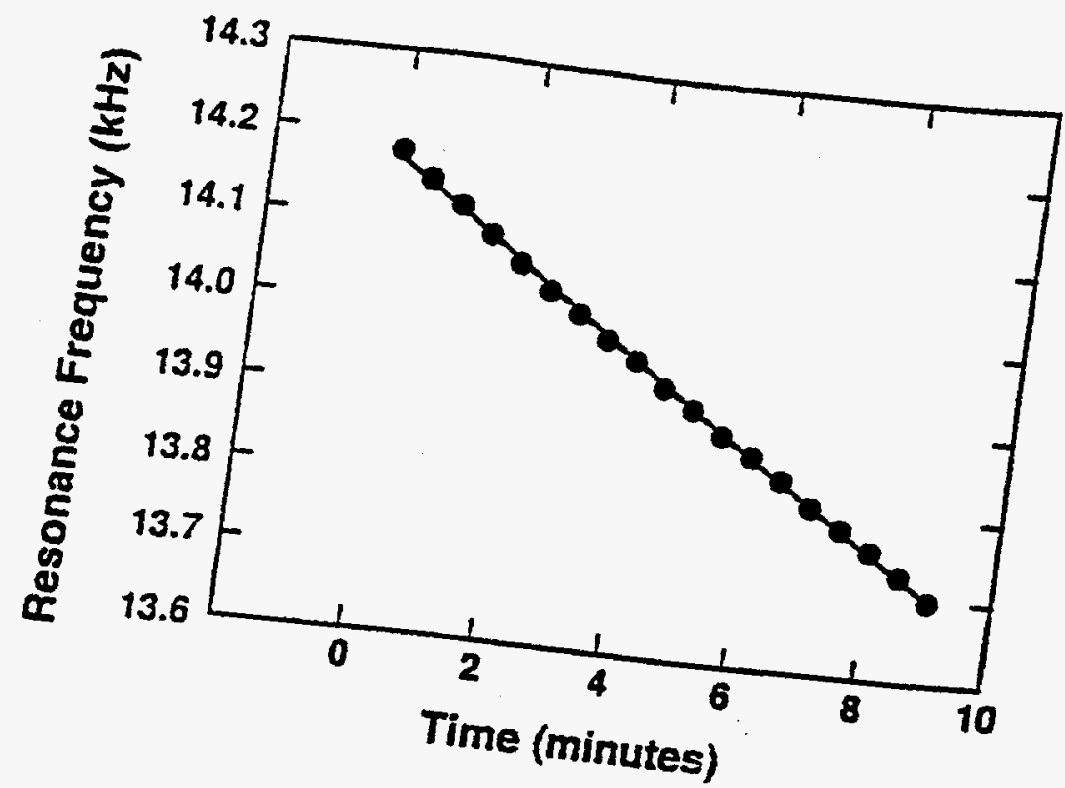

FlG. 4

DISCLAIMER

This report was prepared as an account of work sponsored by an agency of the United States Government. Neither the United States Government nor any agency thereof, nor any of their employees, makes any warranty, express or implied, or assumes any legal liability or responsibility for the accuracy, completeness, or usefulness of any information, apparatus, product, or process disclosed, or represents that its use would not infringe privately owned rights. Reference herein to any specific commercial product, process, or service by trade name, trademark, manufacturer, or otherwise does not necessarily constitute or imply its endorsement, recommendation, or favoring by the United States Government or any agency thereof. The views and opinions of authors expressed herein do not necessarily state or reflect those of the United States Government or any agency thereof. 

. . 